\title{
TRABAJO ADOLESCENTE EN LA EXTRACCIÓN DE MADERA EN LA AMAZONÍA PERUANA: EXPLOTACIÓN LABORAL, TRABAJO FORZOSO, TRATA DE PERSONAS ${ }^{1}$
}

TEENAGER WORKING IN THE EXTRACTION OF WOOD IN THE PERUVIAN AMAZON: LABOR EXPLOITATION, FORCED LABOR, HUMAN TRAFFICKING

TRABALHO ADOLESCENTE NA EXTRAÇÃO DE MADEIRA NA AMAZÔNIA PERUANA: EXPLORAÇÃO DO TRABALHO, O TRABALHO FORÇADO, O TRÁFICO

Jaris Mujica

\section{Resumen}

La extracción de madera es la actividad económica más importante de la Región Ucayali (al este de la Amazonía peruana), aunque un gran porcentaje de esa extracción es ilegal. Esta investigación describe las precarias condiciones de vida y trabajo en los primeros eslabones de la cadena del comercio de madera, y los elementos asociados a la explotación laboral de adolescentes varones, en la dinámica de extracción en caseríos rurales de la cuenca del río Utuquinía (a siete horas de navegación de la ciudad de Pucallpa, capital de la región Ucayali). Sostenida en el análisis de 27 casos (seleccionados de una muestra cuantitativa recogida con una encuesta y complementada con entrevistas), este artículo describe: la función de la mano de obra adolescente en la dinámica de extracción de madera y las condiciones de explotación. Los resultados muestran que la explotación aparece asociada a la precariedad del escenario y a las severas condiciones de inseguridad extendidas en aquellas localidades; y que en un mismo escenario conviven víctimas de explotación, trabajo forzoso y trata de personas.

Palabras clave: Trabajo forzoso, Explotación laboral, Trata de personas, Tala ilegal, Amazonía, Perú. 


\section{Abstract}

Timber extraction is the most important economic activity in the Ucayali region (to the east of the Peruvian Amazon), although a large percentage of that extraction is illegal. This research describes the precarious conditions of life and work in the first clusters in the chain of the timber trade, and items associated with the exploitation of adolescent males in the dynamic extraction in rural villages (in the Utuquinía river, at seven hours of sailing from the city of Pucallpa, capital of the Ucayali region). Based in the analysis of 27 cases of male adolescents (selected from a quantitative sample collected with a survey and complemented with interviews), this article describes the role of adolescent labor in the dynamics of timber extraction and the conditions of exploitation. The results show that the exploitation appears to be associated with the precariousness of the scenario and the severe conditions of insecurity extended in those localities; in this scenario victims of exploitation, forced labor and trafficking persons coexist.

Keywords: Forced labor, Labor exploitation, Human trafficking, Illegal logging, Amazon, Peru.

\section{Resumo}

A extração de madeira é a mais importante atividade econômica na região Ucayali (leste da Amazônia peruana), embora uma grande percentagem dessa extração seja ilegal. Esta pesquisa descreve as precárias condições de vida e de trabalho nos primeiros elos da cadeia do comércio de madeira e itens associados com a exploração de adolescentes do sexo masculino na dinâmica de extração em três aldeias rurais da bacia Utuquinía Rio (sete horas de navegação a partir da cidade de Pucallpa, capital da região de Ucayali). Fundamentada na análise de 27 casos de adolescentes do sexo masculino (selecionados a partir de uma amostra quantitativa coletados em uma pesquisa e suplementados com entrevistas), este artigo descreve a função da mão de obra na adolescência na dinâmica e das condições de operação. Os resultados mostram que a exploração está associado com o contexto precário e as severas condições de insegurança generalizada nessas localidades; em que as vítimas de exploração, trabalho forçado e do tráfico convivem no mesmo espaço.

Palavras-chave: Trabalho forçado, Exploração laboral, Tráfico de pessoas, Extração ilegal de madeira, Peru. 


\section{UNA APROXIMACIÓN CONCEPTUAL: EXPLOTACIÓN LABORAL, TRABAJO FORZOSO, TRATA DE PERSONAS}

La extracción de madera es la actividad económica más importante de Ucayali. Es el principal recurso para la industria regional y también la principal actividad económica de las familias rurales. Sin embargo, hay evidencia de que gran parte de la madera proviene de zonas intangibles (reservas ecológicas) y que se extrae sin permisos de tala (ANDALUZ, 2004; MARONI CONSULTORES, 2006; PEREIRA GONCALVES et al., 2012; EIA, 2012; PÉREZ VILLACORTA, 2014). También hay evidencia de condiciones de pobreza, precariedad y situaciones de trabajo peligroso, explotación laboral, trabajo forzoso y trata de personas en torno a la actividad extractiva (BEDOYA GARLAND y BEDOYA SILVA-SANTISTEBAN, 2005; NOVAK y NAMIHAS, 2009).

El debate conceptual entre aquellas categorías es amplio, y muestra problemas para establecer diferencias operativas, las que se tornan más complicadas si se parte de las definiciones penales o de los ordenamientos jurídicos de cada país. Sin embargo, es posible organizar una aproximación conceptual general a través de un ejercicio heurístico. Una forma amplia de definir la explotación laboral es entenderla como todas las formas de trabajo que vulneran los derechos laborales en relación a los elementos del trabajo decente (salarios bajos, trabajo sin una remuneración, trabajo de horas extra no remuneradas, trabajo sin descanso, etc.) (OIT, 2005). Dentro de la amplia categoría de explotación laboral pueden incluirse formas de trabajo forzoso, y hay casos de explotación laboral consecuencia de la trata de personas (BEATE y HAUCHÈRE, 2009).

La Organización Internacional del Trabajo (OIT, 2005) plantea criterios para definir el trabajo forzoso: este "no puede equipararse con salarios bajos o condiciones laborales precarias, ni abarca solo situaciones de mera necesidad económica" (OIT, 2005, p. 9). Según el Protocolo de la OIT del 2014, que complementa el Convenio nํㅡㄹ sobre el trabajo forzoso de 1930 y el Convenio no105 sobre la abolición del trabajo forzoso de 1957 (ILO, SAP-FL, IPEC, 2012), "se puede considerar trabajo forzoso a todo trabajo o servicio que es exigido a un individuo bajo la amenaza de una pena cualquiera y para el cual no se ofrece voluntariamente". 
Aquella definición implica la existencia de un trabajo o servicio en el que aparezcan dos elementos. i) Que el trabajo o servicio que se exige bajo la amenaza de una pena, cuyos indicadores asociados son: "violencia física contra el trabajador, contra su familia o contra personas cercanas a él", "violencia sexual", "encarcelación u otro confinamiento físico", "penas financieras", "despido del puesto de trabajo", "exclusión de empleos futuros", "exclusión de la comunidad y de la vida social", "supresión de derechos o privilegios", "privación de alimento, cobijo u otras necesidades", etc. ii) Que este el trabajo o servicio se lleve de forma involuntaria. Los indicadores asociados son: "nacimiento en la esclavitud o en la servidumbre", "rapto o secuestro físico", "venta de una persona a otra”, "confinamiento físico en el lugar de trabajo o en detención privada", "coacción psicológica u orden de trabajar acompañada de una amenaza de pena", "endeudamiento inducido", "engaño o falsas promesas sobre el tipo y las condiciones del trabajo", "retención e impago de salarios", "retención de documentos de identidad", etc. (BELSER, DE COCK y MEHRAN, 2005; OIT, 2005; CSI, 2008).

De este modo, el trabajo forzoso comprende situaciones como la esclavitud, prácticas similares a la esclavitud, servidumbre por deudas, etc. (ILO, SAP-FL, IPEC, 2012, p.117-138). Si bien penalmente son fenómenos diferentes, la trata de personas y el trabajo forzoso pueden están directamente relacionados, puesto que la trata -para la explotación laboral, por ejemplo- implica la explotación del trabajo o servicio en condiciones de amenaza de pena y en contextos de no consentimiento o voluntariedad (2012, p.135-136).

En ocasiones el trabajo forzoso se produce como consecuencia de la trata de personas. Aunque es importante indicar que no todo el trabajo forzoso es consecuencia de la trata de personas, "una gran parte de los casos de trata de personas se traducen en trabajo forzoso" (BEATE y HAUCHÈRE, 2009: 5). La trata de personas se define en el Protocolo de Palermo del año 2000 (a través de los elementos explotación, engaño y coerción):

Por trata de personas se entenderá la captación, el transporte, el traslado, la acogida o la recepción de personas, recurriendo a la amenaza o al uso de la fuerza u otras formas de coacción, al rapto, al fraude, al engaño, al abuso de poder o de una situación de vulnerabilidad o a la concesión o 
recepción de pagos o beneficios para obtener el consentimiento de una persona que tenga autoridad sobre otra, con fines de explotación. Esa explotación incluirã, como mínimo, la explotación dela prostitución ajena u otras formas de explotación sexual, los trabajos o servicios forzados, la esclavitud o las practicas análogas a la esclavitud, la servidumbre o la extracción de órganos" (UNODC, 2004: artículo 3).

Para este artículo vamos a utilizar las categorías como conceptos generales que se intersectan (y no en una definición penal específica), de modo que, por ejemplo, algunos casos de explotación laboral se configuran como trabajo forzoso, y algunos casos de trabajo forzoso son consecuencia de la trata de personas. Por ende, utilizamos "trabajo forzoso" como un concepto pivote para el estudio empírico. Esto se justifica porque el concepto se ha plasmado en una propuesta empírica (una herramienta e indicadores desarrollados por la OIT) (OIT, SAP-FL, IPEC, 2012). Se trata de cuestionarios para identificar sujetos en situación de trabajo forzoso a partir de un inventario de condiciones laborales que permiten identificar también situaciones diferentes de explotación laboral y que se pueden operativizar (MUJICA, 2015).

Estos cuestionarios marcan tres dimensiones posibles del trabajo forzoso: i) reclutamiento no libre, ii) vida y trabajo bajo dureza e iii) imposibilidad de dejar al empleador. Cada dimensión tiene indicadores de i) involuntariedad y ii) pena o amenaza de una pena. La aplicación de instrumentos para identificar sujetos víctimas de trabajo forzoso requiere que al menos en una de las dimensiones aparezcan, por lo menos, un indicador de involuntariedad y un indicador de amenaza de pena. La presencia no complementada de indicadores da cuenta de condiciones laborales precarias, pero no necesariamente trabajo forzoso, desde las consideraciones metodológicas propuestas por OIT y que asumimos para esta investigación (MUJICA, 2015). 
Tabla 1. El trabajo forzoso según la confluencia de dimensiones e indicadores

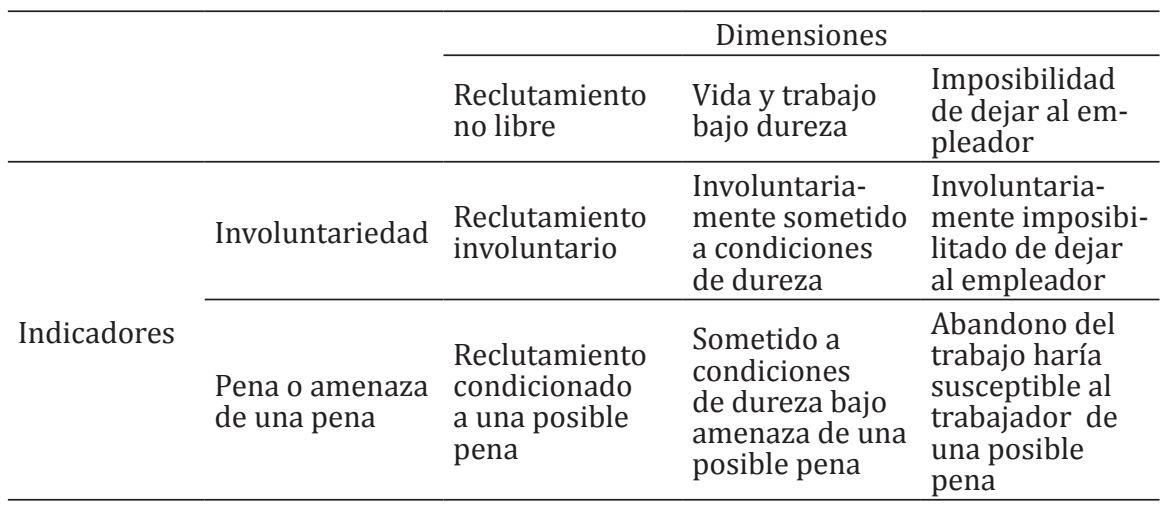

Fuente: Mujica (2015). Elaborado con base a OIT, SAP-FL, IPEC (2012).

\section{OBJETIVOS Y METODOLOGÍA}

Este artículo se basa en los datos recolectados en un trabajo de campo desarrollado en el 2014 (MUJICA, 2015), en el que se buscaba realizar un conteo y caracterización de la explotación laboral y el trabajo forzoso en un escenario maderero. Ahora, el objetivo general de este artículo es describir la situación de explotación laboral en una muestra de varones adolescentes entre 15 y 17 años (sujetos menores de 18 años y pero que estén dentro de la adolescencia tardía) (UNICEF, 2011, p.8) que trabajan en la extracción de madera. Ello implica dos objetivos secundarios: i) determinar cuántos de los adolescentes de la muestra son víctimas de trabajo forzoso; ii) comprender la relación entre las diversas formas de victimización sobre los adolescentes y si alguno es víctima de trata de personas. ${ }^{2}$

Aquello implica dos premisas metodológicas: i) hay una definición de trabajo forzoso que permite identificar el fenómeno en un sujeto victimizado; ii) que el sujeto victimizado lo es en tanto cumple con los indicadores de "involuntariedad" y "pena o amenaza de la pena" en al menos una de las dimensiones ("reclutamiento no libre", "vida y trabajo bajo dureza", "imposibilidad de dejar al empleador") de manera no ocasional.

El proceso de recolección de datos se concentró en la actividad económica de tala de madera, pues es importante para la economía local de 
varias regiones del Perú, y porque se trata de una actividad que recibe escaso control de las autoridades (EIA, 2012). La región Ucayali fue escogida porque es una de las regiones que más cantidad de madera produce (incluyendo la tala ilegal). Se ha recolectado los datos en un espacio que cumplía cuatro características: i) había una comunidad maderera; ii) había uno o más campamentos madereros legales; iii) había uno o más campamentos ilegales; y, iv) existía indicios de trabajo forzoso, determinados por las exploraciones de terreno previas. Se eligió trabajar en los centros poblados del río Utuquinía, en el Distrito de Callería, Provincia de Coronel Portillo en la Región Ucayali, al este de la Amazonía peruana.

\section{Mapa 1. Ubicación del áreaen la que se realizó el trabajo de campo}

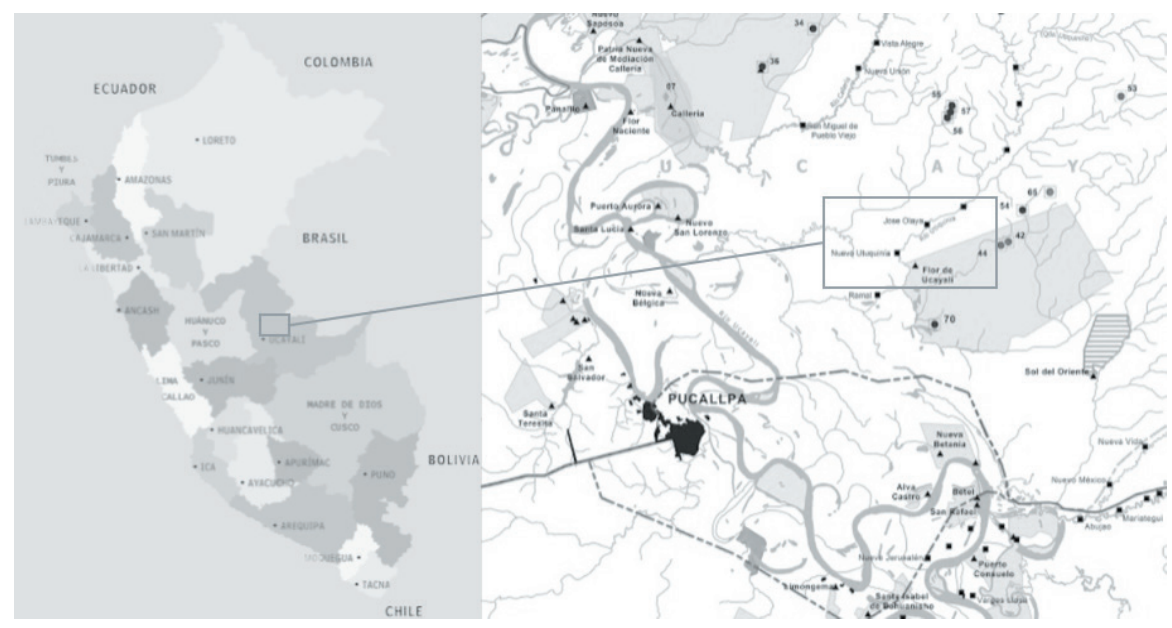

Fuente: Mujica (2015). Detalle de mapas del archivo de la Universidad Católica.

Si bien los datos oficiales muestran que el distrito tiene $14,9 \%$ de pobreza y un IDH de 0,5013 y el 64\% de la población con educación secundaria completa y un ingreso familiar per cápita de US\$210 al mes (INEI, 2012), los datos sobrerepresentan los espacios urbanizados y subrepresentan los centros poblados y caseríos. Sin embargo, los indicadores oficiales presentan datos imprecisos debido al dinero producto de actividades ilegales (ESCOBAR, 2008). El trabajo se concentró en tres centros poblados: 


\section{Tabla 2. Características generales de los centros poblados del trabajo de campo}

\begin{tabular}{ll}
\hline Centro poblado & Características generales \\
\hline Flor de Ucayali & Comunidad nativa Shipibo-Conibo de la margen derecha del Río \\
& $\begin{array}{l}\text { Utuquinía, a } 7 \text { horas de navegación en deslizador de Pucallpa. } \\
\text { Tiene un territorio de 21,290 hectáreas, } 30 \text { habitantes perma- } \\
\text { nentes y una escuela primaria. Hay diversos asentamientos ile- } \\
\text { gales de trabajadores de la madera, y de actividades relacionadas } \\
\text { al narcotráfico. }\end{array}$ \\
\hline Nuevo Utuquinía & $\begin{array}{l}\text { Está distribuido en dos espacios: un centro poblado nuclear y } \\
\text { una zona dispersa de viviendas, campamentos y aserraderos en }\end{array}$ \\
& $\begin{array}{l}\text { ambas orillas del río. Ubicado a } 20 \text { minutos en bote de motor } \\
\text { de Flor de Ucayali, tiene } 50 \text { habitantes permanentes. Tiene una } \\
\text { posta médica y una escuela primaria y secundaria. Tiene una alta } \\
\text { presencia de aserraderos (formales y no formales) y campamen- } \\
\text { tos visibles desde el río. }\end{array}$ \\
\hline A 30 minutos a pie de Nuevo Utuquinía o a 20 minutos por vía \\
fluvial. Con una población de 120 habitantes permanentes tiene \\
un centro poblado y una amplia área dispersa de campamentos y \\
aserraderos. Tiene una escuela primaria.
\end{tabular}

Fuente: Mujica (2015). Elaborado en base a los archivos del Distrito.

El trabajo de campo se realizó en dos periodos, el primero de 21 días en agosto y el segundo de 25 días en octubre del 2014. Se utilizaron tres herramientas: i) encuestas para el conteo y caracterización de la población a través de un muestreo de captura y recaptura (THOMPSON, 2012; WHITFIELD y KELLY, 2002), elaboradas sobre la base del modelo y los criterios desarrollados por la OIT (OIT, SAP-FL, IPEC, 2012). Esto dio como resultado 169 encuestas, 41 de ellas a menores de 18 años, de las cuales analizaremos en este artículo 27 , correspondientes a varones entre 15 y 17 años; ii) entrevistas semiestructuradas a víctimas y trabajadores locales, como a otros actores de la dinámica de extracción de madera, se recolectaron 20 entrevistas, de las cuales analizamos 5, correspondientes al grupo de varones entre 15 y 17 años; iii) observación directa (no participante) de los contextos donde se desarrollan estas actividades, lo que arrojó un amplio cuaderno de campo y un archivo fotográfico.

La observación ha sido la herramienta de acceso a terreno para el equipo de campo (de cuatro investigadores adjuntos) y a través de ella se ha tejido un mecanismo de bola de nieve. El desarrollo del proceso 
de recolección de datos se ha regido con los estándares éticos y de autocuidado expresados los Lineamientos Éticos para la investigación del Laboratorio de Criminología de la Pontificia Universidad Católica del Perú (LABORATORIO DE CRIMINOLOGÍA, 2014). Los datos cuantitativos fueron procesados en tablas de Excel, y los datos cualitativos transcritos fueron analizados en Atlas Ti).

\section{RESULTADOS DEL TRABAJO DE CAMPO}

\subsection{DESCRIPCIÓN GENERAL DEL CONTEXTO DE EXTRACCIÓN DE LA MADERA}

Aunque en Flor de Ucayali existió una concesión legal hasta el 2007, actualmente no se puede explotar legalmente la madera, pues aquel año la comunidad fue multada por extraer madera de zonas prohibidas y se le retiró el permiso. Aún así, la extracción de madera continúa a través de la extracción familiar, ${ }^{3}$ aunque hay zonas en el territorio forestal en el que se ha registrado formas de patronazgo ${ }^{4}$ y dinámicas de peonaje por deudas (BEDOYA GARLAND y BEDOYA SILVA-SANTISTEBAN, 2005; MUJICA, 2015). La comunidad colinda con Nueva Utuquinía y con Santa Sofía, en donde hay sembríos de hoja de coca).

Las comunidades de Nueva Utuquinía y José Olaya tienen, sobre todo, colonos dedicados a la extracción de madera y actividades agrícolas, en algunos casos de hoja coca. Se observa un sistema de patronazgo y, en menor medida, extracción familiar. En estos caseríos se concentran los aserraderos y trabajadores, los que viven en condiciones precarias. Se ha registrado algunos casos de peonaje por deudas a través del adelanto de dinero, la retención de documentos, o retención de paga, en otros casos se realizan préstamos con altos intereses, lo que obliga a los sujetos seguir trabajando hasta saldar sus deudas. Y aunque en el caso de la explotación familiar la coacción no es determinante para el trabajo, esta muchas veces funciona con el reclutamiento de peones para el trabajo complementario.

La mayor parte de los trabajadores es residente de la zona, aunque un grupo pequeño de adultos y dos de los sujetos entre 15 y 17 años 
provienen de centros poblados de los alrededores. Ello se relaciona a las formas de reclutamiento observadas: los sujetos se involucran en la actividad sobre todo a través de su familia, amigos o patrones. La proximidad de la actividad a la vida cotidiana, la dedicación de las familias a la actividad maderera y la transmisión "cara a cara" son factores centrales en este mecanismo de reclutamiento. En todos los casos registrados, los sujetos señalan que su involucramiento obedeció a la necesidad de ganar dinero para apoyar a su familia o solventar gastos domésticos, y la madera representa "la única alternativa para ello" ("la necesidad", "la promesa de ganancia rápida”). En algunos casos, además, se indica que se vinculan a la extracción para producir un capital que, en el futuro, les permita ser patrones y contratar trabajadores.

Es un contexto de precariedad en el que la economía dependede la actividad maderera (sobre todo ilegal) y del complemento económico de la agricultura y pesca (de subsistencia). El trabajo en la madera es una de las pocas actividades que reporta algún tipo de ingreso monetario y que depende de la fuerza de trabajo del individuo, a diferencia, por ejemplo, del transporte que requiere poseer una embarcación o de la agricultura, que requiere una parcela de tierra.

En las comunidades estudiadas el trabajo con la madera de los adolescentes se relaciona a dos eslabones de la cadena de valor: la "extracción de madera" y la "preparación de la madera en los aserraderos". El primer eslabón tiene dos actividades: i) la tala en los bosques, en el territorio estudiado se trata de tala de baja escala en pequeños campamentos, aunque esa madera alimenta el circuito de grandes volúmenes a través de intermediarios; ii) el transporte desde el punto de tala hacia los puntos de preparación, que requiere movilizar grandes troncos por varios kilómetros, sobre todo manualmente, hasta los puntos de transporte fluvial. 


\section{Foto 1. Adolescente y adulto midiendo un tronco recién cortado}

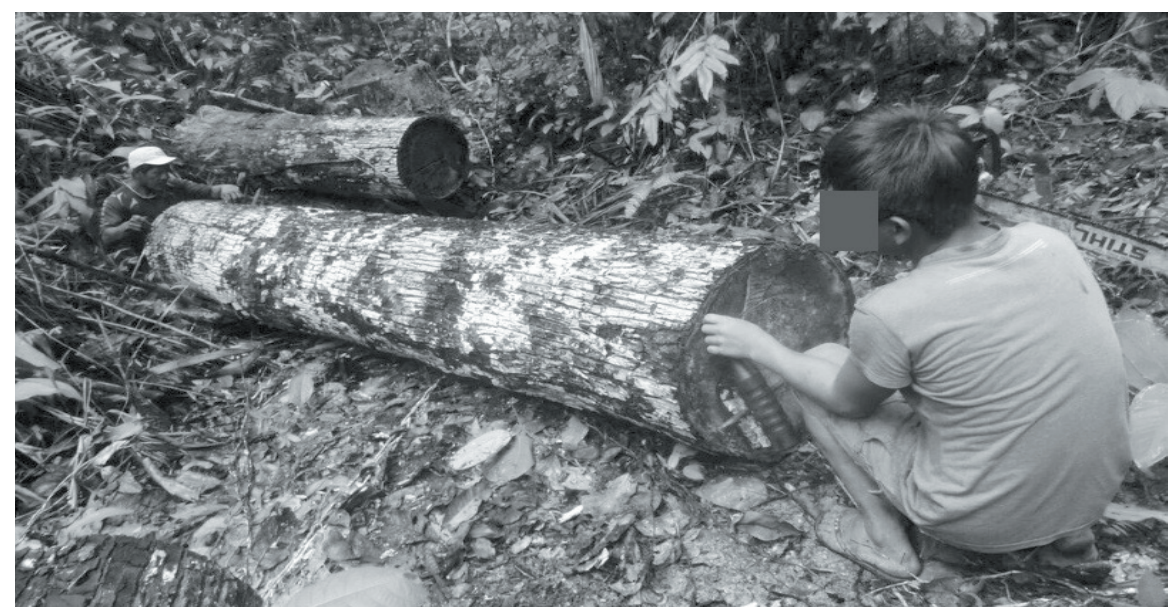

Foto tomada durante el trabajo de campo con un teléfono móvil

El segundo eslabón se divide en dos actividades: i) procesar, mediry cortar la madera, lo que se realiza en pequeños y precarios aserraderos informales situados en las orillas del río; ii) el transporte de la madera del aserradero a los vehículos de transporte fluvial y a la ciudad de Pucallpa.

\section{Foto 2. Adolescentes cargando un tronco}

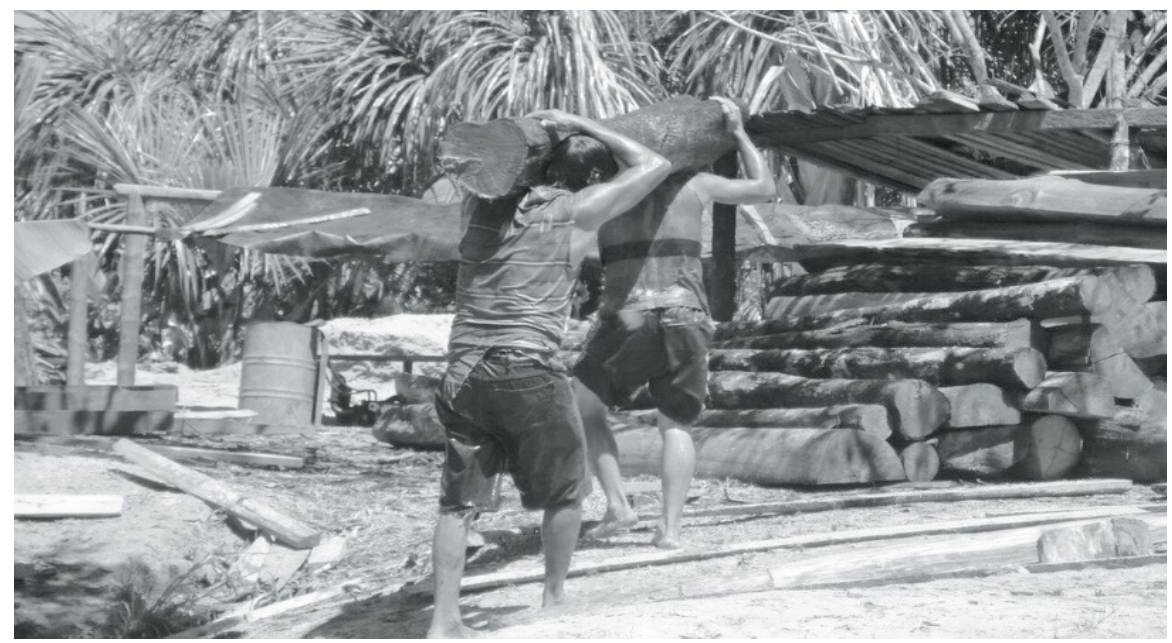

Foto tomada durante el trabajo de campo con un teléfono móvil 


\section{Tabla 3. Actividades y tareas en la explotación de madera en escenario estudiado}

\begin{tabular}{lll}
\hline Eslabón & Actividad & Tareas \\
\hline Extracción & Tala en los bosques & $\begin{array}{l}\text { Matear (despejar de las matas una } \\
\text { zona antes de iniciar la tala) }\end{array}$ \\
\cline { 3 - 3 } & & Tumbar los árboles \\
\cline { 2 - 3 } & & Trozar los árboles \\
\cline { 2 - 3 } & Transporte desde el punto de tala & Cargar la madera hacia el \\
& hacia los puntos de preparación & aserradero \\
\cline { 3 - 3 } & & $\begin{array}{l}\text { Transportar la madera hacia } \\
\text { punto de venta }\end{array}$ \\
\hline Preparación & Procesamiento, medición y corte & $\begin{array}{l}\text { Uso de motosierra para el corte de } \\
\text { madera }\end{array}$ \\
\cline { 2 - 3 } & $\begin{array}{l}\text { Transporte de la madera del } \\
\text { aserradero a los vehículos de } \\
\text { transporte fluvial y a la ciudad de }\end{array}$ & $\begin{array}{l}\text { Cargar la madera hacia las embar- } \\
\text { caciones }\end{array}$ \\
\cline { 2 - 3 } & $\begin{array}{l}\text { Transportar la madera hacia los } \\
\text { Puntos de venta }\end{array}$ \\
\hline
\end{tabular}

Elaboración propia en base a Mujica (2015), con base a la observación y entrevistas.

La observación y las entrevistas muestran que las actividades que involucran el tratamiento directo de la madera son realizadas por sujetos expuestos a diversos riesgos de seguridad (cortes debido al uso de motosierras o contusiones por la caída de árboles, etc.). El transporte de madera hacia las vías fluviales (cargar la madera) es una de las tareas más pesadas, muchas veces realizada de manera colectiva por sujetos mayores y menores de 18 años. Esta actividad tiende a realizase por la noche ("cuando el rio está crecido") y lleva consigo problemas de salud y riesgos por la fuerza invertida para movilizar troncos, además de la posibilidad de picaduras de serpientes e insectos. Las tareas de preparación de la madera en el aserradero tienen riesgos asociados al uso de la maquinaria y el transporte de la madera. En todos los casos las tareas realizadas implican severos riesgos que evidencian condiciones de vida y seguridad precarias. 


\section{Foto 3. Adolescente transportando un tablón cortado}

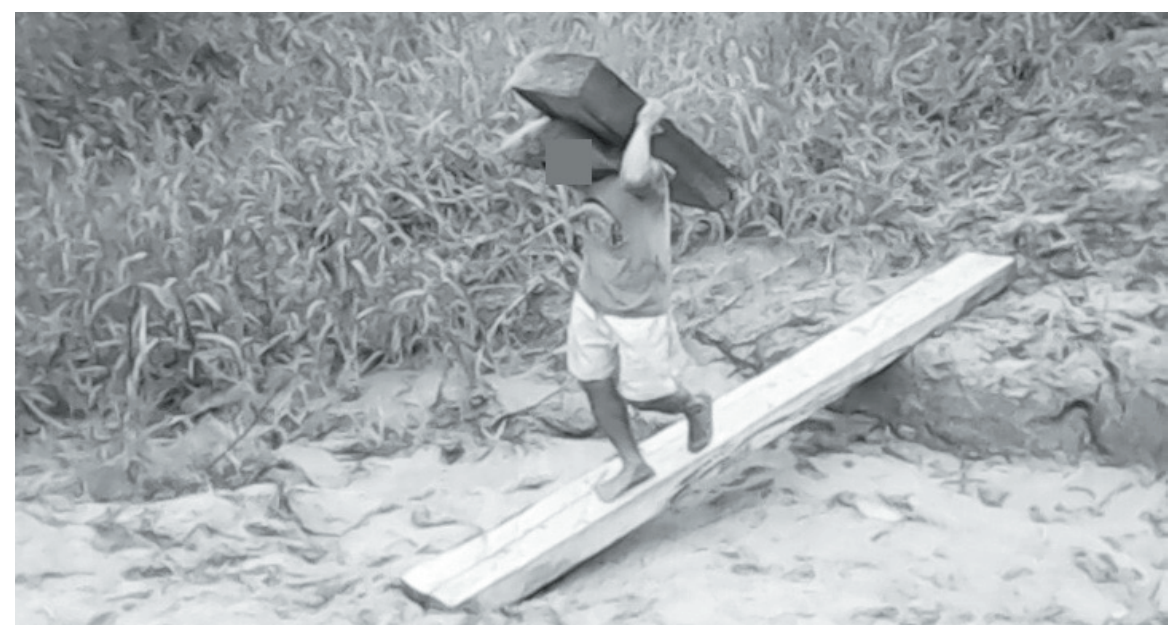

Foto tomada durante el trabajo de campo con un teléfono móvil

\section{TRABAJO DURO Y TRABAJO FORZOSO EN LA EXTRACCIÓN DE MADERA}

El grupo de 27 varones entre 15 y 17 años está dedicado a alguna actividad relacionada a la extracción y preparación de la madera, lo que requiere un alto desgaste energético e implica, en muchos de casos, más de ocho horas de trabajo al día por, al menos, seis días a la semana. Si bien varios declaran recibir un pago por el trabajo realizado, el promedio mensual es considerablemente bajo (más de un tercio del grupo declara menos de US $\$ 100$ al mes) y un tercio declara que no sabe la cantidad de dinero recibido o no recibe una remuneración fija. Esto se debe a que la mayoría no tiene ingresos monetarios, sino en especies, o no tienen una idea clara sobre el esquema de su pago o el dinero es recibido por sus padres, tutores o explotadores directos. 
Tabla 4. Actividades y horas de trabajo declarados por los sujetos entre 15 y 17 años

\begin{tabular}{llcc}
\hline \multirow{3}{*}{ Actividades específicas } & & $\mathrm{n}=27$ & $\%$ \\
\cline { 2 - 4 } & Cortar la madera & 9 & $33.3 \%$ \\
\cline { 2 - 4 } & Cargar la madera & 10 & $37.0 \%$ \\
\cline { 2 - 4 } & Transportar la madera & 8 & $29.6 \%$ \\
\cline { 2 - 4 } & Procesar la madera & 4 & $14.8 \%$ \\
\cline { 2 - 4 } & Almacenar la madera & 3 & $11.11 \%$ \\
\cline { 2 - 4 } & Otros & 3 & $11.11 \%$ \\
\hline Horas de trabajo & Hasta 8 horas & 7 & $25.9 \%$ \\
\cline { 2 - 4 } & Más de 8 horas & 20 & $74.0 \%$ \\
\hline
\end{tabular}

Elaboración propia

El trabajo de campo permitió dar cuenta del panorama del trabajo en el área estudiada y evidencia las condiciones de precariedad extendidas: condiciones paupérrimas de residencia (malocas de madera o estructuras de madera con techos de polietileno o metal prensado), precarias condiciones de educación (la asistencia a la escuela es ocasional y la mayor parte ha abandonado los estudios), precarias condiciones de salud (no hay acceso a una posta médica ni personal de salud, y los adultos de la zona manifiestan tener severas dificultades para acceder a algún tipo de tratamiento o medicamentos). Asimismo, 20 de los sujetos están ligados a formas de explotación familiar (12 de ellos con su familia consanguínea) y 7 a formas de patronazgo.

Hubo un conjunto del grupo que reportaba elementos que se asocian con los indicadores de las dimensiones "vida y trabajo bajo dureza" (92,5\%) y "reclutamiento no libre" (11,1\%), aunque con una concentración evidente en la primera dimensión que ha incluido a los sujetos del segundo grupo. La amplia concentración de sujetos que presentan elementos de la dimensión "vida y trabajo bajo dureza" requiere estudiar dichos elementos asociados a los indicadores de "involuntariedad" y "pena o amenaza de la pena". De ello se desprenden tres datos relevantes: i) el 40,7\% indican que alguna vez le han impedido desplazarse del lugar en el que trabajan (por ejemplo para visitar familiares); ii) el 44,4\% indican haber sido maltratados alguna vez en el trabajo (han recibido golpes por ejemplo); $y$, iii) el 33,3\% indican haber sido amenazados alguna vez en el trabajo. 


\begin{tabular}{|c|c|c|c|}
\hline Indicadores & Elementos registrados & $\mathrm{n}=27$ & $\%$ \\
\hline \multirow[t]{4}{*}{ Involuntariedad } & $\begin{array}{l}\text { Tiene que estar disponible para trabajar } \\
\text { de día y de noche }\end{array}$ & 12 & $44.4 \%$ \\
\hline & $\begin{array}{l}\text { Se le ha obligado a trabajar horas extras } \\
\text { alguna vez }\end{array}$ & 19 & $70.3 \%$ \\
\hline & $\begin{array}{l}\text { Considera que el trabajo que realiza es } \\
\text { peligroso }\end{array}$ & 27 & $100 \%$ \\
\hline & $\begin{array}{l}\text { Alguna vez se le ha impedido desplazarse } \\
\text { o moverse del lugar en el que trabaja (rea- } \\
\text { lizar viajes, visitar a algún familiar, asistir } \\
\text { a la escuela) }\end{array}$ & 11 & $40.7 \%$ \\
\hline \multirow[t]{6}{*}{$\begin{array}{l}\text { Pena o amenaza } \\
\text { de la pena }\end{array}$} & $\begin{array}{l}\text { Hay vigilancia constante durante las horas } \\
\text { de trabajo }\end{array}$ & 9 & $33.3 \%$ \\
\hline & $\begin{array}{l}\text { Hubo retención de algún documento du- } \\
\text { rante el periodo de trabajo }\end{array}$ & 3 & $11.1 \%$ \\
\hline & En alguna ocasión se ha dejado de pagar & 4 & $14.8 \%$ \\
\hline & $\begin{array}{l}\text { En alguna ocasión se ha tenido una lesión } \\
\text { en el trabajo }\end{array}$ & 24 & $88.8 \%$ \\
\hline & $\begin{array}{l}\text { En alguna ocasión se ha recibido maltrato } \\
\text { en el trabajo }\end{array}$ & 12 & $44.4 \%$ \\
\hline & $\begin{array}{l}\text { En alguna ocasión se ha recibido amenazas } \\
\text { en el trabajo }\end{array}$ & 9 & $33.3 \%$ \\
\hline
\end{tabular}

Elaboración propia

La valoración de cada uno de los individuos de que el trabajo que realizan es peligroso (100\%) señala una situación evidente de precariedad, pero debe ser tomada con cautela antes de asociar este elemento a una forma de coacción o control de un tercero. El peligro se relaciona muchas veces de la precariedad del propio contexto y de las dinámicas ilegales de extracción de la madera (la disponibilidad para el trabajo de día o de noche, las horas extras, las lesiones en el trabajo). Sin embargo, hay elementos que se relacionan con más precisión a la explotación de un tercero, aunque aparecen como elementos ocasionales (no implican sistematicidad según la declaración de los individuos): en el indicador de "involuntariedad" aparece el impedimento de movilizarse de su lugar de trabajo (40,7\%); en el indicador "pena o amenaza de la pena" aparece 
el maltrato $(44,4 \%)$ y las amenazas $(33,3 \%)$. Así, aún cuando pueden ser indicadores relevantes para pensar en la posibilidad del trabajo forzoso, estos no aparecen con sistematicidad en el tiempo y podría tratarse de elementos difusos u ocasionales (MUJICA, 2015).

Ahora, incluso si consideramos los elementos ocasionales, es importante entender que son elementos que se cruzan e incluyen entre sí. El dato relevante es que el 33,3\% que ha recibido alguna amenaza implica también al grupo que declara que alguna vez se le ha maltratado y que alguna vez se le ha impedido desplazarse, pero no todos estos han recibido una amenaza algún vez. Esto permite pensar que, al menos, el $33,3 \%$ reportan indicadores de "involuntariedad" y de "pena o amenaza de la pena" de la dimensión "vida y trabajo bajo dureza", aunque se trata de elementos ocasionales, lo que se relaciona con el tipo de actividad, tarea y la dinámica estacional de la extracción de madera. Si bien esto configura la evidencia de una situación de riesgo de trabajo forzoso, por el carácter ocasional, es necesario establecer si se trata de una dinámica constante y una condición del trabajo que configuran una situación efectiva de trabajo forzoso, o se trata de eventos aislados, lo que no se ha podido corroborar en este estudio.

Hay un grupo de sujetos que reporta la retención de documentos $(11,1 \%)$, lo que incluso si fuera una práctica ocasional, es un indicador relevante de trabajo forzoso en la dimensión "vida y trabajo bajo dureza” e, incluso, de trata de personas. Este grupo cumple también con los elementos anteriores y está incluido entre aquellos sujetos que presentan elementos de riesgo de trabajo forzoso, pero no todos los de este grupo reportan retención de documentos. Sin embargo, si estudiamos las otras dimensiones del trabajo forzoso aparece una intersección e inclusión relevante: todos los que reportan "retención de documentos", reportan también indicadores claros de trabajo forzoso en ladimensión "reclutamiento no libre".

Los datos de campo permiten indicar que del grupo de 27 sujetos estudiados al menos 25 (92,5\%) reportan elementos ocasionales de los indicadores de la dimensión "vida y trabajo bajo dureza", lo que pone en evidencia una situación extendida de precariedad y explotación. De este grupo, además de los indicadores ocasionales, al menos 3 sujetos $(11,1 \%)$ 
reportan al mismo tiempo indicadores de la dimensión "reclutamiento no libre". De modo que es posible decir que 3 de los 27 sujetos de la muestra cumplen indicadores de trabajo forzoso.

\section{Tabla 6. Sujetos que reportan indicadores de trabajo forzoso (\%)}

\begin{tabular}{lcc}
\hline Dimensiones & $\mathrm{n}=27$ & $\%$ \\
\hline $\begin{array}{l}\text { Sujetos que reportan elementos ocasionales de los indicadores } \\
\text { de la dimensión "vida y trabajo bajo dureza" }\end{array}$ & 25 & $92.5 \%$ \\
\hline $\begin{array}{l}\text { Sujetos que reportan los indicadores de la dimensión "recluta- } \\
\text { miento no libre" }\end{array}$ & 3 & $11.1 \%$ \\
\hline $\begin{array}{l}\text { Sujetos que reportan los indicadores de la dimensión "imposi- } \\
\text { bilidad de dejar al empleador" }\end{array}$ & 0 & $0 \%$ \\
\hline Sujetos que reportan los indicadores de trabajo forzoso. & 3 & $11.1 \%$ \\
\hline
\end{tabular}

Elaboración propia

\section{DIFERENTES FORMAS DE VICTIMIZACIÓN EN UN MISMO ESCENARIO}

El trabajo de campo permite reportar dos fenómenos relevantes respecto a las formas de explotación de los varones entre 15 y 17 años en el contexto de estudio: i) hay diversas formas de victimización laboral y los sujetos victimizados pueden convivir en el mismo escenario; ii) no hay especialización ni concentración de la misma forma de victimización en un mismo espacio o grupo.

Los datos muestran que los 27 sujetos viven en condiciones precarias, de pobreza y en un contexto de economías de subsistencia. Las actividades y tareas específicas de extracción y preparación de la madera constituyen trabajo peligroso y de alto riesgo para la salud. De este grupo, 25 sujetos presentan elementos ocasionales de los indicadores de la dimensión "vida y trabajo bajo dureza", es decir, amenazas o maltrato ocasional, o que en alguna ocasión se les ha prohibido desplazarse. A su vez de ese grupo, 3 sujetos reportan elementos del indicador "reclutamiento no libre" (además de reportar también elementos ocasionales de "vida y trabajo bajo dureza") y, por lo tanto, encajan en la calificación de "trabajo forzoso". 
Uno de esos sujetos (sujeto 1, de 17 años) ha sido reclutado a través de la "coacción psicológica", o sea, "una orden de trabajar acompañada de una amenaza creíble de pena en caso de incumplimiento" (OIT, 2005, p. 15). Este sujeto realiza sus actividades en un entorno de extracción familiar, cuyo explotador central es el padre de la familia que lo alberga, quien le brinda alimentos y cobijo. Ocasionalmente, los padres de este sujeto, que viven en el mismo caserío, reciben dinero por el trabajo que realiza su hijo y, además, retienen la partida de nacimiento del sujeto 1 . El sujeto 1 asume el trabajo debido a las amenazas expresas de su familia y de su actual explotador (amenazas de ser expulsado de la comunidad, amenazas sobre el uso de la violencia física si no trabaja).

En el caso de los otros dos sujetos (sujeto 2 y sujeto 3, de 16 y 17 años) que encajan en los indicadores de trabajo forzoso, estos realizan sus labores en un mecanismo de patronazgo y hay tres elementos relevantes: de un lado, ambos nacieron y crecieron en comunidades fuera del área de estudio (a dos horas de navegación en el río). Por otro lado, ambos han sido contratados a través de una promesa falsa de trabajo; se les prometió un salario que luego no se pagó y condiciones de trabajo que no eran reales. A ambos se les indicó que trabajarían en la tala de madera cinco días a la semana, recibirían una maloca para dormir y tener sus enseres y objetos personales en la comunidad y recibirían un pago de S/.1200 al mes (alrededor de US\$400). Sin embargo, al llegar al lugar de trabajo el pago no se realizó (reciben un pago ocasional que depende de la cantidad de madera vendida), asimismo, el lugar de residencia no ha sido implementado y deben dormir en el precario campamento en la zona de extracción, trabajan más de ocho horas diarias y al menos seis días a la semana. Además, reciben amenazas constantes, maltrato físico ocasional y han sido expresamente prohibidos de abandonar la zona de trabajo (con la amenaza de castigo físico). Finalmente, el pago que ocasionalmente reciben está enganchado a una deuda asociada a los alimentos que reciben.

En sentido estricto de las definiciones planteadas en la sección 1 de este artículo, los sujetos 2 y 3 son víctimas de trabajo forzoso y también de trata de personas: captación a través de una oferta falsa de trabajo, desplazamiento hacia un lugar de explotación, y explotación a través de formas de coacción. El sujeto 1 podría ser considerado también víctima 
de trata de personas, siempre que se entienda que la cesión de la administración de su cuerpo y fuerza de trabajo haya sido trasladada de sus padres a los explotadores, y se entienda esto como captación y cesión para la explotación. Lo importante, sin embargo, es que las diversas formas de victimización aparecen en el mismo entorno y los sujetos víctimas conviven con otros sujetos que son victimizados de formas diversas o que no son víctimas.

Ahora, ni las formas de explotación laboral asociadas a la dimensión "vida y trabajo bajo dureza", ni los casos de trabajo forzoso identificados corresponden exclusivamente a las formas de patronazgo. Así, al menos una docena de casos de explotación laboral y uno de los casos de trabajo forzoso (sujeto 1) aparecen en dinámicas económicas familiares. De esta manera, tanto en los modos familiares de extracción como en el modo de patronazgo pueden aparecer casos de trabajo forzoso.

De otro lado, los sujetos víctimas de trabajo forzoso no aparecen aislados del resto de sujetos o en un entorno propio: el sujeto 1 vive en un entorno de extracción maderera familiar, y convive con sujetos que presentaban indicadores de la dimensión "vida y trabajo bajo dureza” y con sujetos adultos que no habían sido víctimas de formas de coacción. Lo mismo sucede con los otros dos sujetos víctimas de trabajo forzoso en modos de extracción de patronazgo (sujeto 2 y sujeto 3 ), pues conviven con sujetos que viven y trabajan bajo dureza, pero también con sujetos adultos que han sido víctimas de coacción. En una misma forma de organización económica (explotación familiar o patronazgo) y en un mismo grupo residencial, puede haber sujetos que son víctimas de explotación laboral y otros de trabajo forzoso.

\section{DISCUSIÓN}

Los datos del trabajo de campo muestran la dureza de la vida y el trabajo de los adolescentes en el contexto estudiado, y es en ese marco en el que se registran los casos de trabajo forzoso y los casos de trata de personas. Sin embargo, estos casos no aparecen como mecánicas aisladas de la dinámica de trabajo y de la precaria infraestructura económica del 
entorno, sino más bien como casos extremos en un escenario de pobreza, vulnerabilidad extendidas y desigualdades estructurales. La vida y el trabajo bajo dureza, con evidentes condiciones de explotación y trabajo peligroso, aparecen como parte de la dinámica informal (e ilegal) de la extracción de madera; la distancia respecto a los centros urbanos, la ausencia de autoridades del Estado y la dificultad para denunciar la explotación laboral y otras vulneraciones (debido a la necesidad de ingresos monetarios en un contexto de pobreza y debido a la situación de informalidad e ilegalidad de la extracción) generan un escenario precario y de alto riesgo para el trabajo forzoso y otras formas de victimización.

Aquello permite establecer dos elementos de discusión. Primero, los casos registrados no parecen explicarse por el desarrollo de una organización criminal especializada en la captación o en la explotación de adolescentes para ser utilizados como mano de obra. De este modo, no se detectan dinámicas profesionales de trata de personas para la explotación laboral, y la captación para el trabajo forzoso no muestra una dinámica profesionalizada. El uso de mano de obra adolescente tampoco muestra una dinámica de movilidad geográfica relevante ni traslado de zonas alejadas de los campamentos. Esto refuerza resultados de investigaciones precedentes en la Amazonía peruana (MUJICA, 2013, 2014a, 2014b, 2015; MUJICA y CAVAGNOUD, 2011) y en otras partes del mundo (ARONOWITZ, 2009; BEATE y BELSER, 2009), y muestra algunos contextos en los que las dinámicas de explotación se explican más por la propia precariedad del contexto, la ilegalidad de las dinámicas económicas, la ausencia del Estado o formas de control formal, que por el desarrollo de organizaciones criminales complejas.

Del mismo modo, los casos estudiados no se reportan como un fenómeno nuevo en la zona y no se asocian directamente como consecuencia de la extracción industrial (empresarial) de madera. Se reportan más bien como formas de explotación que existen hace "mucho tiempo" con la novedad de que la extracción de madera se destina actualmente, casi en su totalidad, al núcleo comercial industrial en Pucallpa. Esto permite discutir respecto al uso de formas tradicionales de explotación laboral en nuevos contextos industriales. 
Segundo, los casos estudiados permiten discutir la composición de las dinámicas y formas asociadas a la explotación laboral. De este modo, si bien hay una discusión jurídica que muestra diferencias en torno a los conceptos "trata de personas", "trabajo forzoso" y "explotación laboral”, cuya función normativa es fundamental para el procesamiento de casos (ALLAIN Y HICKEY, 2012) y la composición del derecho positivo; en la práctica, en el territorio estudiado, los casos no aparecen con diferencias evidentes (demográficas o residenciales o de explotación); las diferencias se han podido establecer a través de instrumentos complejos de recolección de datos (demográficos, biográficos y laborales). Estos fenómenos han aparecido en un mismo contexto y con fenomenologías que tienen similitudes, aun cuando pueden ser penalmente diferentes.

Esto sugiere discutir: i) la pertinencia de estos conceptos y la distinción operativa en la construcción de estrategias de focalización para el control del crimen o en estrategias de rescate de víctimas, pues ¿es posible pensar en estrategias focalizadas de rescate de víctimas (de trabajo forzoso o de trata) en contextos precarios como el descrito y en los que hay desigualdades estructurales? (UHL, 2010; MAGAR, 2012; AHMED Y MEENA, 2012; AMAHAZION, 2014); ii) si bien en la discusión conceptual es posible detectar diferencias entre los tipos de víctimas, ¿es posible establecer mecanismos de intervención situacionales solo sobre un tipo de víctima (de trata por ejemplo) aún cuando en contextos de precariedad y desigualdades estructurales hay diversos tipos de victimización en el mismo escenario?; iii) si bien la literatura precedente ha estudiado con rigor las diferencias normativas y penales entre la explotación, la trata de personas y el trabajo forzoso, es también importante estudiar sus similitudes fenomenológicas y la relación empírica entre los casos. Pues si bien es cierto que muchas veces hay confusiones en la catalogación de estos tipos, es posible que esto se deba a la complejidad de la estructura conceptual que produce una "realidad jurídica” (BARATTA, 2004[1982]; O’BRIEN, 2013), cuando en ocasiones estos fenómenos aparezcan juntos en la práctica. 


\section{CONCLUSIONES}

Los datos muestran que los 27 sujetos entre 15 y 17 años que trabajan en la extracción de madera de la muestra estudiada viven en condiciones precarias y realizan un trabajo de alto desgaste energético y con riesgos para la salud. Un grupo de 25 sujetos vive y trabaja en un contexto precario y presenta indicadores ocasionales de trabajo forzoso, y 3 de ellos pueden clasificarse como sujetos víctimas de trabajo forzoso (y víctimas de trata de personas). Estas formas de victimización aparecen como consecuencia de un contexto de precariedad estructural y no como resultado del desarrollo del crimen organizado, de modo que es necesario determinar elementos (demográficos y biográficos) para estudiar porqué en un mismo contexto hay diferentes formas e intensidades de victimización, si estas formas varían en la línea biográfica y si hay modificaciones en las condiciones de explotación de los individuos, y a qué se deben.

\section{NOTAS}

1 Este artículo se sostiene en los datos de campo recolectados en Mujica (2015). La investigación se llevó a cabo en el marco del Proyecto "Consolidando y difundiendo esfuerzos para combatir el trabajo forzoso en Brasil y Perú", financiado por el Departamento de Trabajo de Estados Unidos (USDOL) e implementado a través de la Oficina para los Países Andinos de la Organización Internacional del Trabajo (OIT). El trabajo de campo ha sido posible gracias al apoyo de Teresa Torres, al Laboratorio de Criminología y estudios sobre la Violencia de la Pontificia Universidad Católica del Perú y al trabajo de Nicolás Zevallos, Sofía Vizcarra, Leonidas Ramos, Fanny Pineau y Néstor Paiva.

2 Si bien el Protocolo de Palermo establece una distinción entre la trata de personas en menores de 18 años y la trata de adultos, pues en los menores de 18 años los medios de coerción no son necesarios (BEATE Y HAUCHÈRE, 2009, p. 5), en este artículo vamos a asumir los indicadores generales del trabajo forzoso y la trata de personas para los sujetos entre 15 y 17 años, para poder describir la fenomenología de estas prácticas, identificar los mecanismos de coacción y no las consideraciones penales de estas.

3 Espacios de extracción de madera en donde los sujetos pertenecen a una o varias familias. Estos se asocian ( 3 a 5 personas) para explotar una parcela comunal y ocasionalmente reclutan trabajadores. Las entrevistas indican que no hay una estructura jerárquica definida sino una división de funciones, según la experiencia,y una división sexual y etaria del trabajo. Los campamentos se ubican en puntos cercanos al espacio de extracción ( 1 a 2 horas a pie) lo que permite volver cada día para comer y dormir en la comunidad.

4 Es una modalidad jerárquica con una cabeza (un "patrón o jefe") que tiene a su cargo varios trabajadores. Las entrevistas y la observación muestran campamentos de 4 a 10 trabajadores, en muchos casos en tiendas precarias. La actividad se desarrolla entre las 8 ha y $16 \mathrm{~h}$, pues luego de la jornada los trabajadores locales vuelven a los caseríos en los que residen. Muchas veces el patrón brinda alimentación y un jornal. Las actividades realizadas son informales y las condiciones de trabajo precarias. 


\section{REFERENCIAS}

Andaluz, Carlos. Mecanismos Legales e Institucionales para el Control de la Tala Ilegal de Madera. Lima: Proterra, 2004.

Ahmed, Aziza y Meena Seshu. We Have the Right Not to Be 'Rescued'... When AntiTrafficking Programmes Undermine the Health and Well-Being of Sex Workers. Anti-Trafficking Review, n. 1, 2012. p. 149-65.

Allain, Jean y Hickey, Robin. Property and the Definition of Slavery. International \& Comparative Law Quarterly, vol. 61, n. 4, 2012. p. 915-38.

Amahazion, Fikre. Global Anti-Sex Trafficking: State Variance in Implementation of Protectionist Policies. Human Rights Quarterly, vol. 36, n. 1, 2014. p. 176-209.

Aronowitz, Alexis. Human Trafficking, Human Misery: The Global Trade in Human Beings. Westport, Connecticut, London: Praeger. 2009.

Beate, Andrees y Belser, Patrick. Forced Labor: Coercion and Exploitation in the Private Economy. Boulder, Colorado: Lynne Rienner. 2009.

Baratta, Alessandro. Criminología crítica y crítica del derecho penal: introducción a la sociología jurídico penal. Buenos Aires: Siglo XXI. 2004 [1982].

Beate, Andrees y Hauchère, Aurélie. El trabajo forzoso y la trata de personas: manual para los inspectores de trabajo. Organización Internacional del Trabajo, Programa Especial de Acción para Combatir el Trabajo Forzoso. Ginebra: OIT. 2009. Disponible en: http://www.ilo.org/wcmsp5/groups/public/@ ed_norm/@declaration/documents/publication/wcms_107704.pdf. Última consulta: 1 de abril del 2016.

Belser, Patrick, De Cock, Michaelle y Mehran, Farhad. ILO Minimum Estimate of Forced Labour in the World. 2005. Disponible en: http://www.ilo.org/ wcmsp5/groups/public/---ed_norm/---declaration/documents/publication/ wcms_081913.pdf.Última consulta: 12 de noviembre del 2014.

Bedoya Garland, Eduardo y Bedoya Silva-Santistebán, Alvaro. El Trabajo Forzoso en la Extracción de Madera en la Amazonía Peruana. Lima: Organización Internacional del Trabajo. 2005. 
CSI. Trabajo Forzoso. 2008. Disponible en: http://www.ilo.org/wcmsp5/ groups/public/ed_norm/declaration/documents/publication/wcms_116681. pdf. Útima consulta: 12 de noviembre del 2015.

EIA. La máquina lavadora: Cómo el fraude y la corrupción en el sistema de concesiones están destruyendo el futuro de los bosques de Perú. 2012. Disponible en: http://launderingmachine.files.wordpress.com/2012/04/spanish_report_eia_final2.pdf. Última consulta: 12 de noviembre del 2015.

Escobar, Juan Manuel. Una medición de la Economía Subterránea peruana a través de la demanda de efectivo: 1980 - 2005. Lima: CIES. 2008. Disponible en: http://www.cies.org.pe/es/investigaciones/politica-macroeconomicaycrecimiento. Última consulta: 10 de enero del 2016.

ILO, SAP-FL, IPEC. Hard to see, harder to count: Survey guidelines to estimate forced labour of adoults and children. 2012. Disponible en: http://www.ilo. org/wcmsp5/groups/public/---ed_norm/---declaration/documents/publication/wcms_182096.pdf.Última consulta: 1 de febrero del 2015.

INEI. IV Censo Nacional Agropecuario. 2012. Disponible en: http://proyectos. inei.gob.pe/CenagroWeb/resources/documentos/metodologicos/manualdelRevisor-ivcenagro.pdf.Última consulta: 13 de febrero del 2015.

Laboratorio de Criminología y estudios sobre la Violencia. Lineamientos éticos para la investigación y autocuidado. Lima: Laboratorio de Criminología. 2014.

Magar, Verónica. Rescue and Rehabilitation: A CriticalAnalysis of Sex WorkersAntitraffickingResponse in India. Signs, vol. 37, n. 3, 2012. p. 619-644.

Maroni Consultores. Análisis preliminar sobre legislación en el sector forestal en el Perú. Washington: World Bank.2006.

Mujica, Jaris. Precariedad y trabajo forzoso en la extracción de madera: un estudio en espacios rurales de la Amazonía peruana. Lima: Oficina de la OIT para los Países Andinos. 2015.

Mujica, Jaris. Elementos comparados del impacto de la trata de personas en la salud de víctimas adolescentes en el contexto de la minería ilegal en Madre de Dios. Lima: Promsex, Anesvad, OMS-OPS. 2014. 
Mujica, Jaris. Trayectorias y ciclos de explotación sexual y trata para la explotación sexual de mujeres en la Amazonía peruana. Anthropologica, vol. 33, n. 33. 2014a. p. 163-177.

Mujica, Jaris. The microeconomics of sexual exploitation of girls and Young women in the Peruvian Amazon. Culture, Health \& Sexuality. An International Journal for Research, Intervention and Care, vol. 15, n. 2. 2013. p. 141-152.

Mujica, Jaris y Cavagnoud, Robin. Mecanismos de explotación sexual y trata de niñas y adolescentes en los alrededores del puerto fluvial de Pucallpa. Anthropológica, vol. 23, n. 23. 2013. p. 91-100.

Novak, Fabián y Namihas, Sandra. La trata de personas con fines de explotación laboral: El caso de la minería aurífera y la tala ilegal de madera en Madre de Dios. Lima: IDEI, PUCP. 2009.

O'Brien, Erin. The Politics of Sex Trafficking: A Moral Geography. Critical Criminological Perspectives. Houndmills, Basingstoke, Hampshire: Palgrave Macmillan. 2013.

OIT. Una alianza global contra el trabajo forzoso. 2005. Disponible en: http:// www.ilo.org/wcmsp5/groups/public/---ed_norm/---declaration/documents/ publication/wcms_082334.pdf.Última consulta: 1 de febrero del 2015.

Pereira Goncalves, Marilyne., Panjer, Melissa, Greenberg, Theodorey Magrath, William. Justice for forests. Washington: World Bank. 2012.

Pérez Villacorta, Rosario. Estructura de las redes de corrupción en el proceso de extracción y comercialización de madera extraída de concesiones forestales otorgadas en la provincia de Coronel Portillo, región Ucayali, entre los años 2008 y 2011. Lima: Tesis de Maestría en Ciencia Política de la Pontificia Universidad Católica del Perú. 2014.

Thomson, Steven. Sampling. Londres: Wiley.2012.

Uhl, Bärbel Heide. Lost in Implementation? Human RightsRhetoric and Violations: a CriticalReview of Current European Anti-TraffickingPolicies. Security \& Human Rights, vol. 21, n. 2. 2010. p. 119-126. 
UNICEF. Estado mundial de la infancia. 2011. Disponible en:http://www. unicef.org/honduras/Estado_mundial_infancia_2011.pdf.Últimaconsulta: 4 dejunio del 2015.

UNODC. Protocolo para prevenir, reprimir y sancionar la trata de personas, especialmente mujeres y niños, que complementa la convención de las Naciones Unidas contra la delincuencia organizada transnacional. 2004. Disponible en: http://www.accem.es/ficheros/documentos/pdf_trata/Protocolo_Palermo_-_ESP.pdf. Última consulta: 14 de mayo del 2016.

Whitfield, Kathry y Kelly, Heath. Using the two-source capture-recapture method to estimate the incidence of acute flaccid paralysis in Victoria, Australia. Bulletin World Health Organization, vol. 80, n. 11, 2012. Disponible en: http://www. scielosp.org/scielo.php?script=sci_arttext\&pid=S0042-96862002001100003 Última consulta: 16 de julio del 2015.

Recebido em: 21-7-2016

Aprovado em: 19-12-2016

\section{Jaris Mujica}

Es Candidato a Doctor, Master en Ciencia Política y Licenciado en Antropología por la Pontificia Universidad Católica del Perú (PUCP); es doctorando y Master en Criminología por la Universtitat Pompeu Fabra. Ha sido Jefe de la Unidad de información e inteligencia de la Procuraduría Anticorrupción, Jefe de la Oficina de Asuntos Internos del sistema penitenciario, Comisionado de la Unidad Anticorrupción del Ombudsman en el Perú e investigador para diversas oficinas del sistema de Naciones Unidas. Ha publicado diversos libros y numerosos artículos científicos sobre crimen y violencia en los países andinos, patrones de organización criminal y corrupción. Actualmente es profesor del Departamento de Ciencias Sociales e Investigador Principal del Laboratorio de Criminología de la PUCP. E-mail: imujica@pucp.edu.pe

Pontificia Universidad Católica del Perú. Departamento de Ciencias Sociales Av. Universitaria 1801. San Miguel. Lima 32. Peru. 